\title{
Hyper-acute neurogenic pulmonary oedema in a prehospital setting: a case report
}

\author{
Lucija Galič ${ }^{1, \star}$, Andrej Fabjan²,3, Vesna Homar ${ }^{1,2}$
}

${ }^{1}$ Community Health Centre Vrhnika, 1360 Vrhnika, Slovenia

${ }^{2}$ Faculty of Medicine, University of Ljubljana, 1000 Ljubljana, Slovenia ${ }^{3}$ Department of Neurology, University Medical Centre Ljubljana, 1000

Ljubljana, Slovenia

\section{*Correspondence}

lucija.galic@zd-vrhnika.si

(Lucija Galič)

\begin{abstract}
Introduction: Neurogenic pulmonary oedema (NPO) is a form of pulmonary oedema which can develop after an acute central nervous system (CNS) lesion, most often a subarachnoid haemorrhage (SAH). Its pathogenesis is associated with a surge of catecholamines which are released into the systemic circulation following an abrupt increase in intracranial pressure or a strategic CNS lesion. NPO typically presents with dyspnoea, tachypnoea, hypoxia, pink frothy sputum, bilateral crackles on auscultation, and bilateral infiltrates on chest X-ray in a few hours or days following the event.

Case report: The following case report describes a case of hyper-acute NPO following a SAH, which presented as acute respiratory failure in the minutes after the event and required fast and aggressive treatment in the prehospital setting.

Conclusion: The management of NPO is primarily focused on prompt diagnosis and treatment of a CNS event and supportive care.
\end{abstract}

\section{Keywords}

Pulmonary oedema; Prehospital emergency care; Neurology

\section{Introduction}

Neurogenic pulmonary oedema (NPO) is defined as hypoxemic respiratory failure shortly after a catastrophic central nervous system (CNS) event or strategic CNS lesion [1]. It follows a subarachnoid haemorrhage (SAH) in up to $25 \%$ of cases [2], and is also seen with intracerebral and subdural haemorrhage, stroke in the insula or brainstem, traumatic brain or spinal cord injury, epilepsy, and meningitis [1,3-5]. NPO usually presents with dyspnoea, tachypnoea, hypoxia, pink frothy sputum, bilateral crackles on auscultation, and bilateral infiltrates on chest X-ray, and usually resolves within 24 to 72 hours with appropriate management $[1,3]$.

The pathophysiology of NPO is poorly understood, but there are several proposed mechanisms [1,3]. An abrupt increase in intracranial pressure or a strategic CNS lesion (such as in the insula or dorsolateral medulla) can cause a sudden systemic surge of catecholamines [3-5]. This can lead to reversible myocyte injury and segmental wall motion abnormalities, impaired heart pumping function, elevated cardiac enzymes, ECG changes, and arrhythmias, albeit with no clinical evidence of an acute myocardial infarction [1, 3, 6-8]. A catecholamine-induced abrupt increase in systemic arterial pressure increases the left ventricle afterload, which can lead to pulmonary congestion and hydrostatic transudative pulmonary oedema $[1,3]$. Increased pulmonary capillary pressure can damage the pulmonary endothelium, resulting in vascular leak and exudative pulmonary oedema $[1,3]$. A CNS injury is also related to a systemic inflammatory response, with release of pro-inflammatory cytokines that can further aggravate lung injury and oedema $[1,2,7]$. Autonomic nervous system activation in CNS events is related to atrial tachyarrhythmias such as atrial fibrillation (AF), which can impair the cardiac pumping function and further contribute to the development of NPO [8].

NPO can lead to acute cardiopulmonary failure with global hypoperfusion and hypoxia leading to severe secondary ischemic brain damage or death $[6,7,9,10]$. For this reason, NPO usually warrants emergent intensive care unit (ICU) management of pulmonary oedema and treatment of the underlying CNS event $[1,7]$.

In this article we report a case of hyper-acute NPO due to an aneurysmal subarachnoid haemorrhage, which required prompt and aggressive treatment beginning in a prehospital setting.

\section{Case report}

The patient was a 59-year old nurse with a history of arterial hypertension and Hashimoto's thyroiditis. She had undergone a cardiac catheter ablation due to atypical atrioventricular nodal re-entrant tachycardia three years before, and was taking $4 \mathrm{mg}$ of perindopril daily.

The patient developed a headache with general malaise, tachycardia and palpitations while in her workplace, and a few hours later she suddenly lost consciousness. The prehospital emergency medicine team (PEMT), consisting of an emergency physician and three paramedics, responded to the 
TA B L E 1. The patient's vital signs at time of arrival of PEMT, immediately after intubation, and on arrival at hospital.

\begin{tabular}{lccc} 
& 0 min & 4 min & 43 min \\
& On arrival & After RSI & In hospital \\
Respiratory rate (breaths per minute) & 5 & 15 & 10 \\
Heart rate (beats per minute) & 180 & 170 & 115 \\
Blood pressure (mmHg) & Unmeasurable & $132 / 76$ & $138 / 91$ \\
\hline Oxygen saturation (\%) & Unmeasurable & 82 & 89 \\
Consciousness & Coma & Sedated & Sedated \\
\hline End tidal $\mathrm{CO}_{2}(\mathrm{mmHg})$ & $/$ & 26 (hyperventilated with BVM) & 32 \\
\hline
\end{tabular}

PEMT, prehospital emergency medicine team; RSI, rapid sequence intubation; BVM, bag valve mask.

emergency call and arrived at the scene in eight minutes. Upon arrival, the patient was unresponsive to any stimuli, and had constricted, unreactive pupils. There was pink frothy sputum in her mouth, and diffuse bilateral crackles on lung auscultation. The patient was breathing agonally with a rate of 5 breaths per minute, and had a palpable pulse over the carotid artery. Arterial blood pressure and oxygen saturation were unmeasurable. The 3-lead ECG monitor showed AF with a rate of 180 beats per minute.

The PEMT performed a rapid sequence intubation (RSI) with fentanyl $100 \mathrm{mcg}$ IV, etomidate $20 \mathrm{mg}$ IV and suxamethonium $100 \mathrm{mg} \mathrm{IV}$, and the patient was initially ventilated in IPPV mode with $100 \%$ oxygen with a frequency of 10 breaths per minute and a minute ventilation of 6 litres per minute. Because of the clinical signs of pulmonary oedema the patient was administered furosemide $40 \mathrm{mg}$ IV. She received minimal fluid resuscitation of approximately $250 \mathrm{~mL}$ of crystalloid before admission to hospital. At admission her haemoglobin $(\mathrm{Hb})$ was $155 \mathrm{~g} / \mathrm{L}$. The patient's vital signs are shown in Table 1.

Once stable, the patient was transported to a Division of Neurology 43 minutes after the first contact with the PEMT. On admission, computed tomography (CT) of the head showed an extensive subarachnoid haemorrhage at the base of the brain, and CT angiography of the cerebral arteries showed a ruptured aneurysm of the anterior communicant artery (Fig. 1). A chest X-ray showed signs of moderate to severe pulmonary oedema with bilateral alveolar and interstitial infiltrates (Fig. 2).

The patient underwent immediate endovascular coiling of the aneurysm (Fig. 3) and was transferred to neurological ICU, where she remained under treatment for 35 days. The patient was ventilated with IPPV mode, PEEP $8 \mathrm{~cm} \mathrm{H}_{2} \mathrm{O}$, a frequency of 8 breaths per minute and tidal volume of $560 \mathrm{~mL}$. Oxygen supplement was reduced from $100 \%$ to $30 \%$ the second day. Diuresis was spontaneous and transiently increased due to furosemide and mannitol given for pulmonary and cerebral oedema. Fluid balance was maintained. With supportive care, the pulmonary oedema had regressed almost completely on day 2 after the event (Fig. 2). In the first 14 days she required high doses of vasoactive support with noradrenaline and argipressin, and inotropic support with dobutamine. An ECG showed sinus rhythm with frequent paroxysmal AF. She had a cardiac index (CI) of $3.5 \mathrm{~L} / \mathrm{min} / \mathrm{m}^{2}$ and elevated levels of troponin I up to $14,000 \mathrm{ng} / \mathrm{L}$ (positive at $>40 \mathrm{ng} / \mathrm{L}$ ), which be-
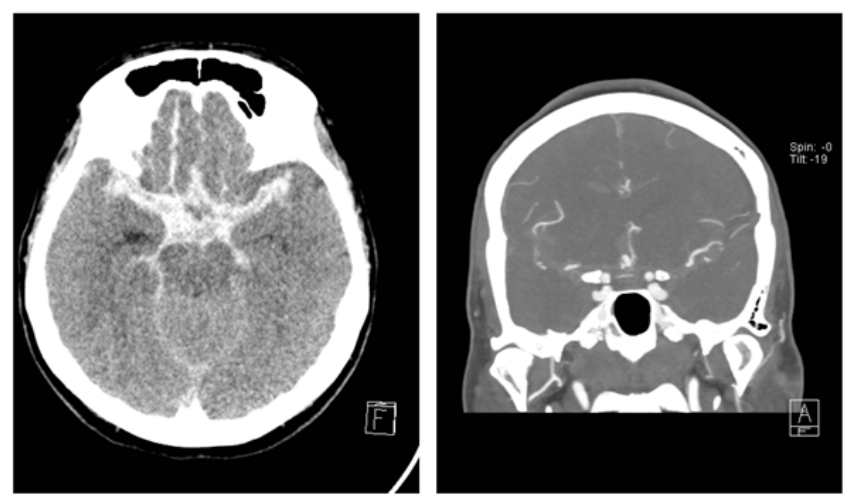

FIGURE 1. Head CT (left) and CTA (right) at time of admission to a Division of Neurology (CT-Computed Tomography, CTA-CT Angiography).

gan to decrease on the second day. Echocardiography showed no signs of segmental wall motion abnormalities. After three weeks she was gradually taken off analgosedation; she was breathing spontaneously with a percutaneous tracheostomy, and began to open her eyes spontaneously and to verbal stimuli, without any other meaningful contact.

\section{Discussion}

This case demonstrates that, unlike the usual presentation in hours to days, NPO can develop within minutes and can cause hyper-acute respiratory failure. On-field differential diagnoses included both cardiogenic and neurogenic causes of the patient's sudden loss of consciousness, so the prehospital treatment was symptomatic. Nevertheless, respiratory failure was successfully managed by early RSI and on-field mechanical ventilation in an attempt to reverse the pulmonary oedema and hypoxia.

The patient received intravenous loop diuretic furosemide as a symptomatic treatment of pulmonary oedema. Diuretic therapy is often used in SAH patients with pulmonary oedema, even though the oedema is not of cardiac origin [7]. However acute impairment of cardiac function was probably present in our patient, as demonstrated subsequently by reduced CI, elevated troponin levels and the need for inotropic and vasoactive support in hospital. Additionally, the patient was in $\mathrm{AF}$ which could be triggered by the SAH and could contribute to the development of NPO. Therefore our case could most 

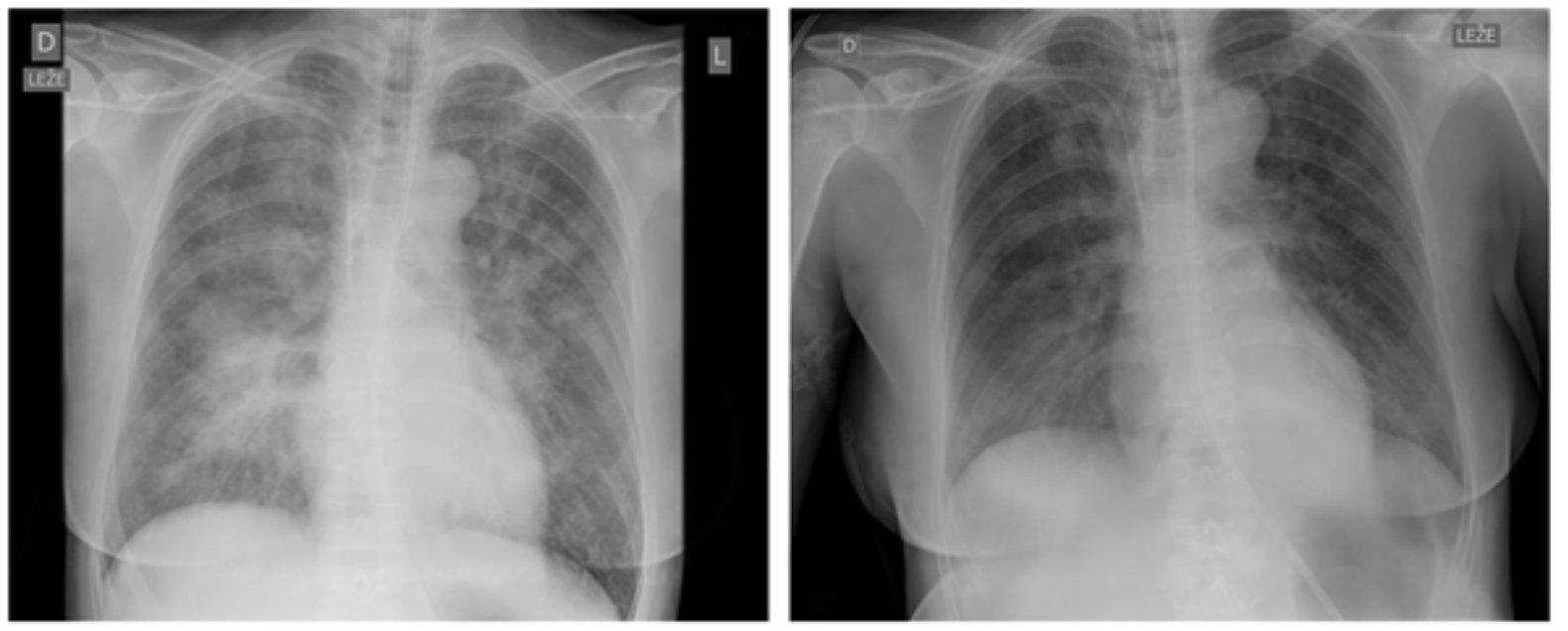

F I G U R E 2. Chest X-ray at time of admission to a Division of Neurology (left) and the next day (right).

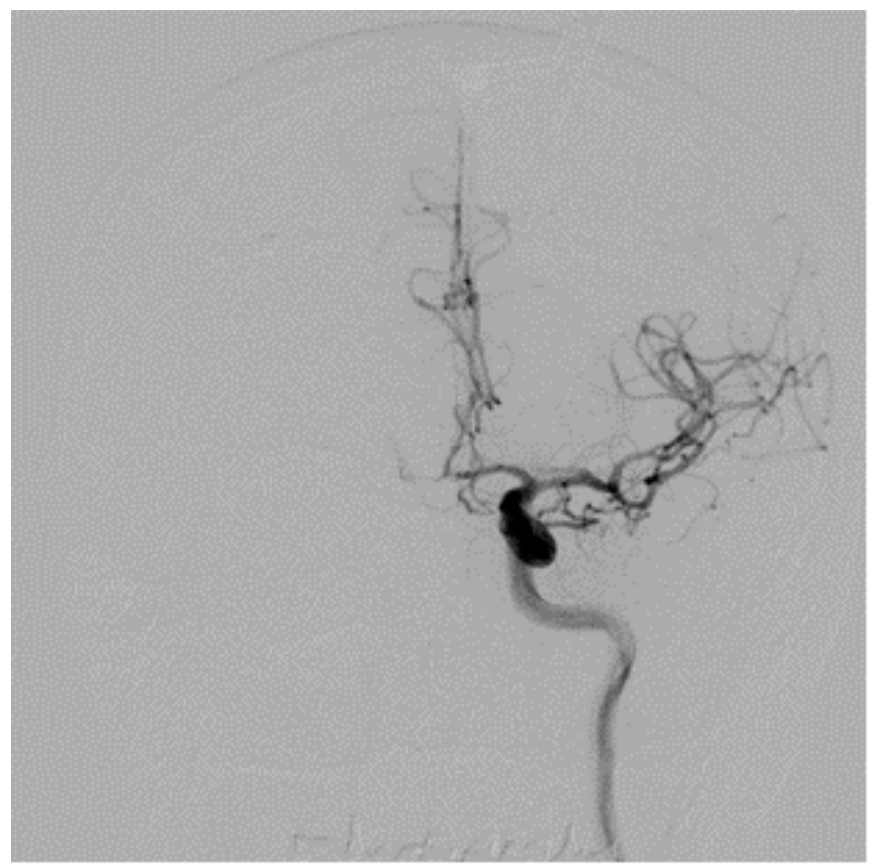

F I GURE 3. DSA after endovascular coiling of the aneurysm (DSA-Digital Subtraction Angiography).

comprehensively be described as a cardiogenic neurogenic oedema, as it was a consequence of an unfortunate cascade of events, beginning with a neurological condition which had a detrimental effect on the patient's cardiac function. It should be noted that some authors suggest urgent $\alpha$-(phentolamine) and $\beta$-adrenergic (propranolol) blockade, as this could decrease catecholamine-related myocardial injury [7]. On the other hand, it is interesting that $\beta$-stimulating catecholamines, especially dobutamine, also improve cardiac function in NPO $[7,10]$.

It should also be noted that diuretics can aggravate hypovolemia and add a hypovolemic contribution to the cardiogenic shock. In an emergency setting, therefore, it is a challenge to estimate the need for fluid resuscitation. Due to the unknown volume status and clinical signs of pulmonary oedema, the use of emergency diuretics was a reasonable choice. Since the patient's $\mathrm{Hb}$ was slightly elevated upon arrival at hospital, it points towards a justified use of a diuretic, since increased $\mathrm{Hb}$ matches increased extravascular lung water content. In such cases the use of point-of-care ultrasonography could help towards an optimal fluid resuscitation in the field.

The ratio of the dead space ventilation $(\mathrm{Vd})$ to tidal volume $(\mathrm{Vt})(\mathrm{Vd} / \mathrm{Vt})$ by expired $\mathrm{CO}_{2}$ (volumetric capnometry) was not available prior to admission to the ICU. This would contribute to a more precise estimation of ventilation-perfusion disturbances. The severe ventilation-perfusion disturbance was demonstrated by severe hypoxemia and acted upon in the field.

\section{Conclusions}

In some cases, NPO can take a hyper-acute course, leading to respiratory failure and secondary ischemic brain injury. This case encourages emergency physicians to consider NPO in patients with diminished consciousness and respiratory failure without an obvious cardiac or pulmonary condition. Early analgesia, sedation, relaxation, endotracheal intubation and proper ventilation are crucial in treating any pulmonary oedema in a prehospital setting, but prompt diagnosis and treatment of a CNS event can further improve the patient's management and outcome.

\section{AUTHOR CONTRIBUTIONS}

LG and VH conceived of the presented manuscript. LG wrote the manuscript with support from $\mathrm{VH}$ and AF. AF contributed mostly with the hospital course of diagnosis and treatment. All authors contributed to the final version of the manuscript. $\mathrm{VH}$ supervised the project.

\section{ETHICS APPROVAL AND CONSENT TO PARTICIPATE}

Research was approved by National Medical Ethics Committee of Republic of Slovenia. Reference number of the approval: 0120-533/2020-7. 


\section{ACKNOWLEDGMENT}

The authors thank to Institute for development of Family Medicine for their support of the project and to all the peer reviewers for their opinions and suggestions.

\section{FUNDING}

This research received no external funding.

\section{CONFLICT OF INTEREST}

The authors declare no conflict of interest.

\section{REFERENCES}

[1] Baumann A, Audibert G, McDonnell J, Mertes PM. Neurogenic pulmonary edema. Acta Anaesthesiologica Scandinavica. 2007; 51: 447455.

[2] Junttila E, Ala-Kokko T, Ohtonen P, Vaarala A, Karttunen A, Vuolteenaho $\mathrm{O}$, et al. Neurogenic pulmonary edema in patients with nontraumatic intracerebral hemorrhage: predictors and association with outcome. Anesthesia \& Analgesia. 2013; 116: 855-861.

[3] Davison DL, Terek M, Chawla LS. Neurogenic pulmonary edema. Critical Care. 2012; 16: 212.
[4] Cheung RT, Hachinski V. The insula and cerebrogenic sudden death. Archives of Neurology. 2000; 57: 1685-1688.

[5] Raja HM, Herwadkar AV, Paroutoglou K, Lilleker JB. Neurogenic pulmonary oedema complicating a lateral medullary infarct. BMJ Case Reports. 2018; 2018: bcr2018225437.

[6] Bruder N, Rabinstein A. Cardiovascular and pulmonary complications of aneurysmal subarachnoid hemorrhage. Neurocritical Care. 2011; 15: 257-269.

[7] Macmillan CSA, Grant IS, Andrews PJD. Pulmonary and cardiac sequelae of subarachnoid haemorrhage: time for active management? Intensive Care Medicine. 2002; 28: 1012-1023.

[8] Chen P, Chen LS, Fishbein MC, Lin S, Nattel S. Role of the autonomic nervous system in atrial fibrillation: pathophysiology and therapy. Circulation Research. 2014; 114: 1500-1515.

[9] Fontes RBV, Aguiar PH, Zanetti MV, Andrade F, Mandel M, Teixeira MJ. Acute neurogenic pulmonary edema: case reports and literature review. Journal of Neurosurgical Anesthesiology. 2003; 15: 144-150.

[10] Muroi C, Keller M, Pangalu A, Fortunati M, Yonekawa Y, Keller E. Neurogenic pulmonary edema in patients with subarachnoid hemorrhage. Journal of Neurosurgical Anesthesiology. 2008; 20: 188-192.

How to cite this article: Lucija Galič, Andrej Fabjan, Vesna Homar. Hyper-acute neurogenic pulmonary oedema in a prehospital setting: a case report. Signa Vitae. 2021;17(4):208211. doi:10.22514/sv.2021.087. 